\title{
THE SECOND HOMOTOPY GROUP OF SPUN 2-SPHERES IN 4-SPACE
}

\author{
BY J. J. ANDREWS AND S. J. LOMONACO ${ }^{1}$
}

Communicated by R. H. Bing, May 27, 1968

1. Introduction. Andrews and Curtis [1] have shown that the second homotopy group of the complementary domain of a locally flat 2-sphere $S^{2}$ in the 4-sphere $S^{4}$ may not be trivial. This was shown to be the case if $S^{2}$ is formed by spinning the trefoil knot. Epstein [3] has shown that if $S^{2}$ is a spun nontrivial 2-sphere, then $\pi_{2}\left(S^{4}-S^{2}\right)$ is a free abelian group of infinite rank. Fox [6] has suggested that it might be more fruitful to consider the second homotopy group with its $\pi_{1}$-action, and has asked for an algorithm for calculating $\pi_{2}\left(S^{4}-S^{2}\right)$ as a $J \pi_{1}$-module. Sumners [8] has constructed a knotted 2-sphere in $S^{4}$ for which $\pi_{2}$ has nontrivial $J \pi_{1}$-torsion.

The following theorem gives the structure of $\pi_{2}$ as a $J \pi_{1}$-module for the case of spun 2-spheres.

THEOREM 2. If $k\left(S^{2}\right) \subset S^{4}$ is a 2-sphere formed by spinning an arc $A$ about the sphere $S^{2}$ and $\left(x_{0}, x_{1}, \cdots, x_{n}: r_{1}, r_{2}, \cdots, r_{m}\right)+$ is a presentation of $\pi_{1}\left(S^{4}-k\left(S^{2}\right)\right)$ with $x_{0}$ the image of the generator of $\pi_{1}\left(S^{2}-A\right)$ under the inclusion map, then

$$
\left(X_{i}(1 \leqq i \leqq n): \sum_{i=1}^{n}\left(\partial r_{j} / \partial x_{i}\right) * X_{i}=0(1 \leqq j \leqq m)\right)
$$

is a presentation of $\pi_{2}\left(S^{4}-k\left(S^{2}\right)\right)$ as a $J \pi_{1}$-module.

2. Outline of proof. Let $S^{n}$ be the standard $n$-sphere. Let $S_{ \pm}^{n}$ be the closed domains of $S^{n}-S^{n-1}$. Let $A$ be an arc in $S_{+}^{3}$ which meets $S^{2}$ only in the end-points of $A$. Now rotate $S_{+}^{3}$ about $S^{2}$. Then $A$ sweeps out a 2-sphere $k\left(S^{2}\right)$ called a spun 2-sphere [2].

THEOREM 1. If $k\left(S^{2}\right) \subset S^{4}$ is a spun 2-sphere, then $\pi_{2}\left(S^{4}-k\left(S^{2}\right)\right)$ $\simeq K /[K, K]$, where $K$ is the kernel of the homomorphism $i_{*}: \pi_{1}\left(S^{3}-k\left(S^{2}\right)\right)$ $\rightarrow \pi_{1}\left(S^{4}-k\left(S^{2}\right)\right)$ induced by inclusion and $[K, K]$ is the commutator subgroup of $K$.

1 Supported by NSF Grant GP-5458. 
Proof in brief. Let $[f] \in K$, where $f:\left(S^{1}, 1\right) \rightarrow\left(S^{3}-k S^{2}, p\right)$. Since [f] lies in the kernel of $i_{*}$, there exists a $g:\left(S^{2}, 1\right) \rightarrow\left(S^{4}-k S^{2}, p\right)$ such that

(1) $g \mid S^{1}=f$,

(2) $g\left(S_{+}^{2}\right) \subset S_{+}^{4}-k S^{2}$,

(3) $g\left(S_{-}^{2}\right) \subset S_{-}^{4}-k S^{2}$.

Define $\Phi: K \rightarrow \pi_{2}\left(S^{4}-k\left(S^{2}\right)\right)$ as $\Phi[f]=[g]$. It follows from the asphericity of knots [7] that $\pi_{2}\left(S_{ \pm}^{4}-k\left(S^{2}\right)\right)=0$, and hence that $\Phi: K$ $\rightarrow \pi_{2}\left(S^{4}-k\left(S^{2}\right)\right)$ is a well-defined homomorphism. It can now be shown that $\Phi$ is onto and has $[K, K]$ as its kernel.

Note that the following sequences are exact:

$$
\begin{gathered}
1 \rightarrow K \stackrel{j_{*}}{\rightarrow} \pi_{1}\left(S^{3}-k\left(S^{2}\right)\right) \stackrel{i_{*}}{\rightarrow} \pi_{1}\left(S^{4}-k\left(S^{2}\right)\right) \rightarrow 1 \\
1 \rightarrow[K, K] \rightarrow K \rightarrow \pi_{2}\left(S^{4}-k\left(S^{2}\right)\right) \rightarrow 0 .
\end{gathered}
$$

Hence the action of $\pi_{1}\left(S^{4}-k\left(S^{2}\right)\right)$ on $\pi_{2}\left(S^{4}-k\left(S^{2}\right)\right)$ is obtained by lifting the elements of $\pi_{1}\left(S^{4}-k\left(S^{2}\right)\right)$ by $i_{*}$ to $\pi_{1}\left(S^{3}-k\left(S^{2}\right)\right)$ and then applying the natural action of $\pi_{1}\left(S^{3}-k\left(S^{2}\right)\right)$ on its normal subgroup $K$.

Let $\left(x_{0}, x_{1}, x_{2}, \cdots, x_{n}: r_{1}, r_{2}, \cdots, r_{m}\right)^{\phi}$ be a presentation of $\pi_{1}\left(S^{4}-k\left(S^{2}\right)\right)$ with $x_{0}$ representing the image of the generator of $\pi_{1}\left(S^{2}-k\left(S^{2}\right)\right)$ under the homomorphism $j_{*}: \pi_{1}\left(S^{2}-k\left(S^{2}\right)\right)$ $\rightarrow \pi_{1}\left(S^{4}-k\left(S^{2}\right)\right)$ induced by inclusion. A corresponding presentation of $G=\pi_{1}\left(S^{3}-k\left(S^{2}\right)\right)$ is $\left(x_{0}, x_{ \pm 1}, x_{ \pm 2}, \cdots, x_{ \pm n}: r_{ \pm 1}, r_{ \pm 2}, \cdots, r_{ \pm m}\right)^{\phi}$, where $r_{i}\left(x_{0}, x_{-1}, \cdots, x_{-n}\right)=r_{-i}$. Then $K$ is the normal closure of $\left\{\phi\left(x_{i} x_{-i}^{-1}\right)\right\}$ in $G$.

By means of the Reidemeister-Schreier theorem [5] it can be shown that:

LeMma 7. $\left(\left\{\boldsymbol{x}_{\alpha}\right\}_{\boldsymbol{\alpha} \in H}:\left\{\boldsymbol{r}_{\boldsymbol{\alpha}}\right\}_{\alpha \in H},\left\{x_{-i \beta}(i \geqq 0)\right\}_{\beta \in H}\right)$ is a presentation of $K$, where $H=\pi_{1}\left(S^{4}-k\left(S^{2}\right)\right)$.

Lifting the action of $\pi_{1}\left(S^{4}-k\left(S^{2}\right)\right)$ on $\pi_{2}\left(S^{4}-k\left(S^{2}\right)\right)$ up to this presentation, we have Theorem 1 .

\section{REFERENCES}

1. J. J. Andrews and M. L. Curtis, Knotted 2-spheres in the 4-sphere, Ann. of Math. (2) 70 (1959), 565-571.

2. Emil Artin, Zur Isotopie zweidemensionaler Flachen im $R^{4}$, Abh. Math. Sem. Univ. Hamburg 4 (1925), 174-177.

3. D. B. A. Epstein, Linking spheres, Proc. Cambridge Philos. Soc. 56 (1960), 215-219.

4. R. H. Fox, Free differential calculus. I: Derivation in the free group ring, Ann. of Math. (2) 57 (1953), 547-560. 
5. - Free differential calculus. III: Subgroups, Ann. of Math. (2) 64 (1956), $407-419$.

6. - Some problems in knot theory, Topology of 3-manifolds symposium, Prentice-Hall, Englewood Cliffs, N. J., 1962.

7. C. D. Papakyriakopoulos, On Dehn's lemma and the asphericity of knots, Ann. of Math. (2) 66 (1957), 1-26.

8. D. L. Sumners, $O n \pi_{2}$ of $S^{2}$ in $S^{4}$ (to appear).

Florida State University, Tallahassee, Florida 32306

\title{
CLASSIFICATION OF KNOTS IN CODIMENSION TWO
}

\author{
BY RICHARD K. LASHOF AND JULIUS L. SHANESON
}

Communicated by W. Browder, May 31, 1968

Introduction. In this paper we consider smooth knots, i.e., smooth embeddings $\phi: S^{n} \rightarrow S^{n+2}, n \geqq 3$. Two knots $\phi$ and $\eta$ are said to be equivalent if there is a diffeomorphism $f: S^{n+2} \rightarrow S^{n+2}$ such that $f \phi\left(S^{n}\right)=\eta\left(S^{n}\right)$. The embedding $\phi$ extends to an embedding $\phi: S^{n} \times D^{2}$ $\rightarrow S^{n+2}$, and any two such extensions are ambient isotopic relative to $S^{n} \times 0$. Hence if $A=\operatorname{cl}\left(S^{n+2}-\bar{\phi}\left(S^{n} \times D^{2}\right)\right)$, the pair $(A, \partial A)$ is determined up to diffeomorphism by the equivalence class of $\phi$. We call $(A, \partial A)$ the complementary pair, or simply the complement, of the knot $\phi$. In this paper we show that if $\pi_{1} A$, the fundamental group of the knot, is infinite cyclic, then there is at most one knot inequivalent to $\phi$ with complementary pair $(B, \partial B)$ of the same homotopy type as $(A, \partial A)$. This result is of interest because for any $n \geqq 3$ there are many inequivalent knots $\phi: S^{n} \rightarrow S^{n+2}$ with fundamental group $Z$, see for example [12]. (The result also holds in the P.L. case, provided $\phi$ extends to a P.L.-embedding $\bar{\phi}: S^{n} \times D^{2} \rightarrow S^{n+2}$.)

1. Knots with diffeomorphic complements. In [4], Gluck showed that homeomorphisms of $S^{2} \times S^{1}$ are isotopic if and only if they are homotopic and used this result to conclude that there are at most two knots $\phi: S^{2} \rightarrow S^{4}$ with homeomorphic exteriors. In [1], W. Browder studied the pseudo-isotopy classes of diffeomorphisms (and P.L. equivalences) of $S^{1} \times S^{n}$ for $n \geqq 5$. He showed that two P.L. equivalences are pseudo-isotopic if and only if they are homotopic. For the group $D\left(S^{n} \times S^{1}\right)$ of pseudo-isotopy classes of diffeomorphisms, he obtained the exact sequence 